\title{
BMJ Open Study protocol for the Cities Changing Diabetes programme: a global mixed- methods approach
}

\author{
A. David Napier, ${ }^{1}$ John J Nolan, ${ }^{2}$ Malene Bagger, ${ }^{3}$ Louise Hesseldal, ${ }^{3}$ \\ Anna-Maria Volkmann ${ }^{4}$
}

To cite: Napier AD, Nolan JJ, Bagger M, et al. Study protocol for the Cities Changing Diabetes programme: a global mixedmethods approach. BMJ Open 2017;7:e015240. doi:10.1136/ bmjopen-2016-015240

- Prepublication history for this paper is available online. To view please visit the journal online (http://dx.doi.org/10. 1136/bmjopen-2016-015240).

Received 2 December 2016 Revised 24 September 2017 Accepted 28 September 2017

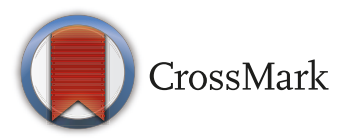

${ }^{1}$ Department of Anthropology, University College London, London, UK

${ }^{2}$ Steno Diabetes Center, Copenhagen, Denmark

${ }^{3}$ Health Advocacy, Cities

Changing Diabetes Programme, Novo Nordisk, Bagsvaerd,

Hovedstaden, Denmark

${ }^{4}$ Department of Clinical,

Educational and Health

Psychology, University College

London, London, UK

Correspondence to

Dr A. David Napier;

d.napier@ucl.ac.uk

\section{ABSTRACT}

Introduction Urban living has been shown to affect health in various ways. As the world is becoming more urbanised and almost two-thirds of people with diabetes now live in cities, research into the relationship between urban living, health and diabetes is key to improving the lives of many. The majority of people with diabetes have type 2 diabetes, a subset linked to overweight and obesity, decreased physical activity and unhealthy diets. Diabetes has significant consequences for those living with the condition as well as their families, relationships and wider society. Although care and management are improving, complications remain common, and diabetes is among the leading causes of vision loss, amputation, neuropathy and renal and cardiovascular disease worldwide. We present a research protocol for exploring the drivers of type 2 diabetes and its complications in urban settings through the Cities Changing Diabetes (CCD) partnership programme.

Methods and analysis A global study protocol is implemented in eight collaborating CCD partner cities. In each city, academic institutions, municipal representatives and local stakeholders collaborate to set research priorities and plan implementation of findings. Local academic teams execute the study following the global study protocol presented here. A quantitative Rule of Halves analysis obtains measures of the magnitude of the diabetes burden, the diagnosis rates in each city and the outcomes of care. A qualitative Diabetes Vulnerability Assessment explores the urban context in vulnerability to type 2 diabetes and identifies social factors and cultural determinants relevant to health, well-being and diabetes. Ethics and dissemination The protocol steers the collection of primary and secondary data across the study sites. Research ethics board approval has been sought and obtained in each site. Findings from each of the local studies as well as the result from combined multisite (global) analyses will be reported in a series of core scientific journal papers.

\section{INTRODUCTION}

Today, $54 \%$ of the world's population lives in areas which have, by custom or definition, come to be referred to as 'urban'. ${ }^{1}$ Urban residence is estimated to increase to $66 \%$ in 2050 , while the global population is forecasted to increase by 2.5 billion over the same period. ${ }^{1}$

\section{Strengths and limitations of this study}

Innovative, large-scale, in-depth data collection based on quantitative and qualitative methodologies.

- Diverse research sites contribute to global (overall) study findings while maintaining local research autonomy and ensuring relevance of findings.

- Study data from each city will not be absolutely comparable because of slightly differing local research needs and data collection/analysis strategies.

- Multisite research study involves data collection in five languages (Danish, Spanish, English, Italian, Mandarin, including various local dialects), meaning that especially qualitative data relies on accurate translation practices into study language (English).

- Studies are needed that compare rural versus urban, emerging mega cities versus declining urban economies, economically unequal versus equal settings.

Much of the increase will be in developing countries, where the rate of urbanisation is most rapid. ${ }^{2}$ Demographic shifts from rural to urban areas are associated with many alterations including changes in public hygiene, sanitation and access to healthcare and in occupational, dietary and physical activity patterns. ${ }^{3}$ These changes have fundamental implications for the emergence of non-communicable diseases, ${ }^{3}$ such as type 2 diabetes.

Worldwide, 415 million adults are living with diabetes, corresponding to $8.8 \%$ of the global adult population. ${ }^{4}$ By 2040 , the number is expected to rise to 642 million. ${ }^{4}$ Diabetes has considerable impact on mortality, disability as well as the lives of affected individuals and their families and presents a substantial cost for society. Diabetes-related costs are a significant economic burden and in 2015 were estimated to account for $12 \%$ of global healthcare expenditure or US\$673 billion. If projections hold true, cost will increase to over US $\$ 802$ billion by $2040 .{ }^{4}$ Approximately $90 \%$ of all people with diabetes have type 2 
diabetes, the development of which is impacted by risk factors such as excess body weight, physical inactivity, poor nutrition, ethnicity, family history of diabetes and increasing age. ${ }^{4}$

Almost two-thirds of all people with diabetes live in areas described as 'urban', and this is expected to increase to $74 \%$ by $2040 .{ }^{4}$ Thus, it is important to identify which characteristics of urban living impact health and well-being and to understand how they contribute to diabetes vulnerability or resilience. Some characteristics of urban living may increase the risk of disease, while others may offer protection $;^{5}$ however, complex synergies of risk factors make it difficult to predict the exact impact of single factors in isolation. Furthermore, cities are complex and dynamic entities and each differs considerably in population size, density, growth rate and so forth.

Most cities present a wide range of economic and social advantages and challenges, ${ }^{5}$ resulting in social and spatial inequalities ${ }^{6}$ that may also reflect broader national contexts. Differences between and within cities make it challenging to generalise on how urban life influences health. An example of this diversity of impact is shown in a study estimating multivariate adjusted ORs of known potential factors associated with diabetes in different income countries. ORs of having diabetes while living in urban areas were 1.07 in high-income countries $(\mathrm{P}=0.6511), 1.20$ in upper-middle-income countries $(\mathrm{P}=0.0930), 1.20$ in lower-middle-income countries $(\mathrm{P}=0.0284)$ and 1.65 in low-income countries $(\mathrm{P}<0.0001){ }^{7}$ Interestingly, conventional risk factors such as age, body mass index (BMI), physical activity, diet, education and family history of diabetes did not fully account for the higher urban prevalence in low-income countries. ${ }^{7}$ Thus, the authors hypothesised that social and cultural factors may be primary drivers of diabetes and those may compound the risk for the disease. ${ }^{7}$

In the context of this study, we differentiate between social factors and cultural determinants and draw on the general discourse around the social determinants of health and their role in creating health inequalities. ${ }^{8-10}$ Traditionally, the social determinants of health encompass the structural conditions in a society, or, in other words, the "wider set of forces and systems shaping the conditions of daily life'. ${ }^{11}$ These forces and systems include, among others, economic policies and systems, development agendas, social policies and political systems which are commonly linked to notions of inequity and societal and individual health inequalities. ${ }^{11}$

Cultural determinants, on the other hand, are in fact often the foundation from which social factors emerge; for local practices and assumptions (at the individual, community or institutional levels) determine in part how social circumstances are created in the first place and, therefore, made sense of locally. By using the term 'cultural determinants', we do not suggest that national, racial, ethnic or religious orientations determine health outcomes, but rather that the values, shared assumptions and conventional practices of groups determine the ethical, moral and behavioural foundations on which individual and collective decisions about health are made.

For example, in settings where gender inequality is a defining cultural feature of local society, a variety of social factors (eg, education level, access to work, economic equality and so forth) are also present and expressed and acted on accordingly. We, therefore, use the term culture in this protocol to refer to shared systems of value (both overt and covert) and their related conventions shared by groups of people in a given social context. Attitudes and beliefs, as well as ways of acting in and on one's environment, are rooted in culture. Relating culture to human health and well-being makes it easier to understand how institutions, communities and individuals respond to a variety of factors that are of an environmental, geographical, political or economic nature.

\section{Rationale}

There is a good knowledge base regarding the impact of biomedical and some socioeconomic factors relevant to diabetes. What has not yet been comprehensibly examined is how these factors interact with cultural and wider social components to mediate both the prevention and the experience of diabetes (and its precursors as well as complications) in urban settings, especially regarding the diagnostic process and long-term care provision.

There remains, in other words, a paucity of empirical studies that identify and examine the complex sociocultural factors relevant to urban diabetes and that integrate those factors into the wider biomedical context of diabetes treatment and prevention. What this means is that until now we have lacked an evidence base for the impact of lived experience on diabetes vulnerability and patterns of morbidity. This absence, we argue, has also prevented us from understanding local prevention and treatment needs, as well as local barriers to adherence. To address this paucity, we employ qualitative data collection and analysis methods directly based on, and linked to, quantitative data generated as part of the same study.

\section{Aims and objectives}

The research protocol presented here is designed to:

1. identify any gaps in the diagnosis and treatment pathways in a given research site through a quantitative Rule of Halves ( $\mathrm{RoH})$ analysis;

2. identify and explore vulnerabilities in a local population as they relate to health, well-being and diabetes through a qualitative Vulnerability Assessment (VA);

3. establish a local evidence base in each study site by synthesising findings;

4. explore the impact of social factors and cultural determinants and their effect on individuals' lives as well as diabetes vulnerability in specific urban settings around the world.

The protocol is designed for the Cities Changing Diabetes (CCD) programme, a cross-disciplinary and cross-sector public/privatepartnership established in 2014 that follows recommendations from the 2013-2020 
WHO Global Action Plan on Non-Communicable Diseases.

Research for the CCD programme is ongoing and conducted from 2015 until 2020. The programme aims to generate an understanding of the burden of diabetes and its social and cultural drivers in a growing number of partner cities. Academic institutions in each partner city generate site-specific evidence available to local city governments and stakeholders for building targeted interventions that can effectively address local drivers of diabetes. Local findings are shared within the programme and made available at a global level for other cities and stakeholders to build on.

The programme consists of three interrelated phases, which are described below.

Mapping: In this first phase, the city-specific diabetes burden and its sociocultural drivers are mapped, either using existing public health data or through a household survey. To date, eight cities are participating in the programme: Mexico City, Houston, Copenhagen, Shanghai and Tianjin were part of a first group and have completed the mapping phase; Vancouver and Johannesburg joined in 2016 and Rome in 2017. The three most recent cities to join the programme are currently in the mapping phase. Additional cities will participate by 2018 . A body of collective knowledge about diabetes and its drivers in the participating cities is being generated.

Sharing: The results from the participating cities are continuously shared with city governments, academics, businesses, charitable organisations and others with an interest in healthy city planning and infrastructure, public health policies, and implementing novel interventions.

Action: Identifying and scaling up solutions to reduce the rate of diabetes in cities is the programme's ultimate goal. In each city, local partners work together to develop and implement city-specific interventions based directly on the research results from the mapping phase. Such actions are expected also to inform cities other than those currently participating.

The global partnership consists of University College London (UCL), Steno Diabetes Center Copenhagen (SDCC) and Novo Nordisk (NN). UCL is the global academic lead for the overall research framework and advises local academic partners in designing and implementing research protocols. SDCC contributes expertise in diabetes clinical epidemiology, registry studies and health promotion research. NN provides the programme management and has committed to fund the programme with US $\$ 20$ million towards 2020. Cities interested in the CCD programme are encouraged to reach out to the global partnership.

Partner cities in the programme are each represented by a local partners group that consists of city government(s), patient organisation(s), provider business(es) and academic institution(s). The total number of local partners varies from city to city. Each local partners group is responsible for local programme design and execution, setting the research framework, promoting diabetes advocacy and risk awareness and communication. Progress in programme implementation is discussed within the local partners groups and in dialogue with global partners and other participating cities through regular face-to-face and online meetings.

\section{METHODS AND ANALYSIS}

Although many studies suggest a complex association between urban living and non-communicable diseases (both beneficial and detrimental to health), most of the published literature has not explored how and why cities affect health. ${ }^{5}$ The complexity of causation and the diversity of mechanisms that may explain how characteristics of urban environments affect the health of people living there require large-scale, cross-disciplinary research focused on shaping population health. ${ }^{5}$

The research protocol presented in this paper, therefore, employs mixed-methods strategies to address this complexity and to explore the breadth and depth of the current status of diabetes in various cities around the globe. The quantitative research component consists of the $\mathrm{RoH}$ analysis and the qualitative research component, the VA, which together provide the foundation of the CCD research framework (figure 1).

\section{Rule of Halves}

The aim of the quantitative research is to generate an overview of the diabetes burden in each city and identify the greatest challenges in relation to diagnosis and/ or treatment. This aim is achieved by estimating the so-called RoH (figure 2). ${ }^{12} 13$ The $\mathrm{RoH}$ is a theoretical framework used in various chronic diseases to measure levels of access to clinical care, adherence and effects of treatment. The first element of the RoH was suggested in the USA in 1947 for non-insulin-dependent diabetes ${ }^{14}$ and in 1964 confirmed in the UK. ${ }^{15}$ The second element of the RoH for diabetes was added in $1976,{ }^{16}$ and in 1980, the third element was established. ${ }^{17}$ The RoH still largely holds true for many other chronic diseases. ${ }^{18}$ For this research, two additional elements are added to the RoH for a total of five, stating that half the people with diabetes are not diagnosed, half of those diagnosed are not receiving care, half of those receiving care are not achieving treatment targets and half of those achieving treatment targets are not achieving the desired outcome (no complications).

\section{Local RoH}

Depending on available city data, local academic partners agree with the local partners group and the global partners which methodology is optimal for establishing a local RoH baseline. The RoH can be estimated either through existing published research or by implementing new research including collecting blood samples, such as was the case in CCD partner Mexico City. The methodologies and results from each of the local RoHs will be published separately. 


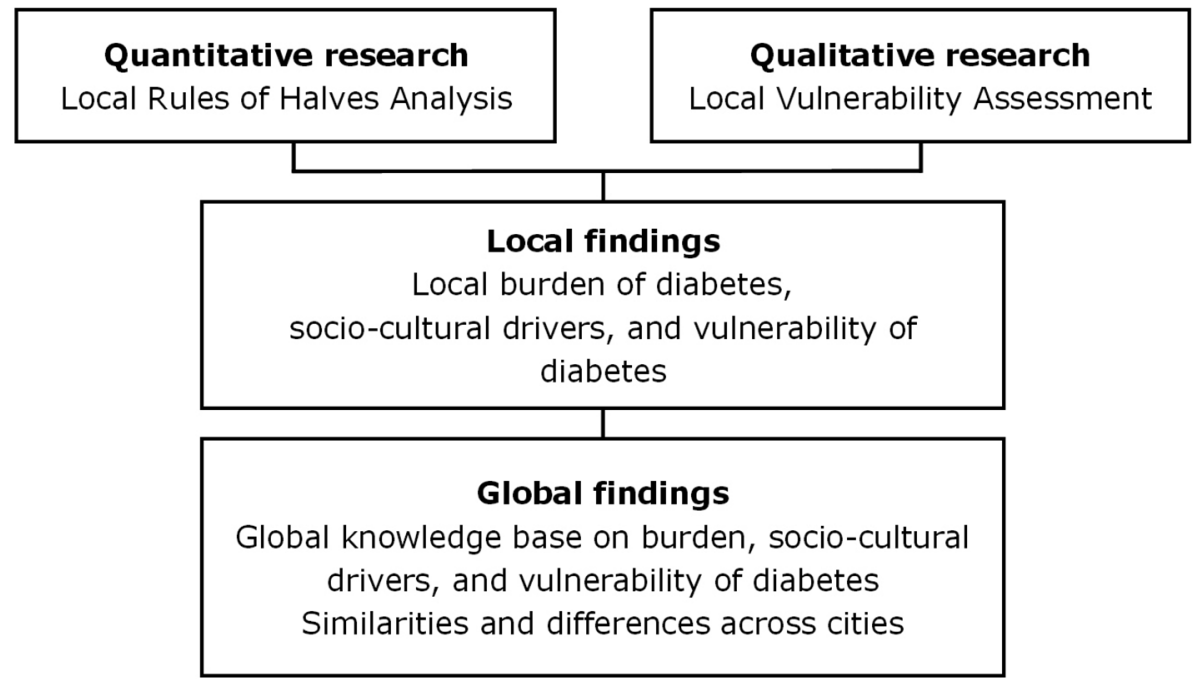

Figure 1 The research framework.

\section{Diabetes Vulnerability Assessment}

The aim of the qualitative research is to study the specific social and cultural drivers that affect residents and that increase or decrease their diabetes vulnerability. The qualitative research is based on an established VA instrument developed previously by the academic lead at UCL in collaboration with the United Nations. ${ }^{19}$ At the foundation of a VA is the idea that addressing vulnerability depends on understanding the specific policy, social, cultural and individual conditions that impact life and well-being in any given field site. Regardless of the site and context of application, the VA examines: the combined effects on individual lives of social and environmental stressors; service use patterns and barriers; community structures; networks and adaptations (forms of social and cultural 'capital') and barriers to human agency and to social and cultural inclusion (vulnerability indicators).
Data that characterises the complex ways in which vulnerability emerges is gathered in each of these areas, making it possible to identify levers that either increases risks or ameliorate their impact.

For the CCD programme, a global Diabetes Vulnerability Assessment (D-VA) was designed by UCL based on the original VA instrument. The use of the D-VA improves understanding of the social, cultural and environmental characteristics that enhance or limit optimal health outcomes in relation to diabetes. The D-VA is a locally sustainable intervention tool because it builds on existing links to communities and stakeholders and creates new networks. The D-VA follows a set procedure (figure 3) and examines three general domains of inquiry: an external formal domain (assessing public assistance and programmes and use thereof); a local community domain (assessing local situations, responses and forms

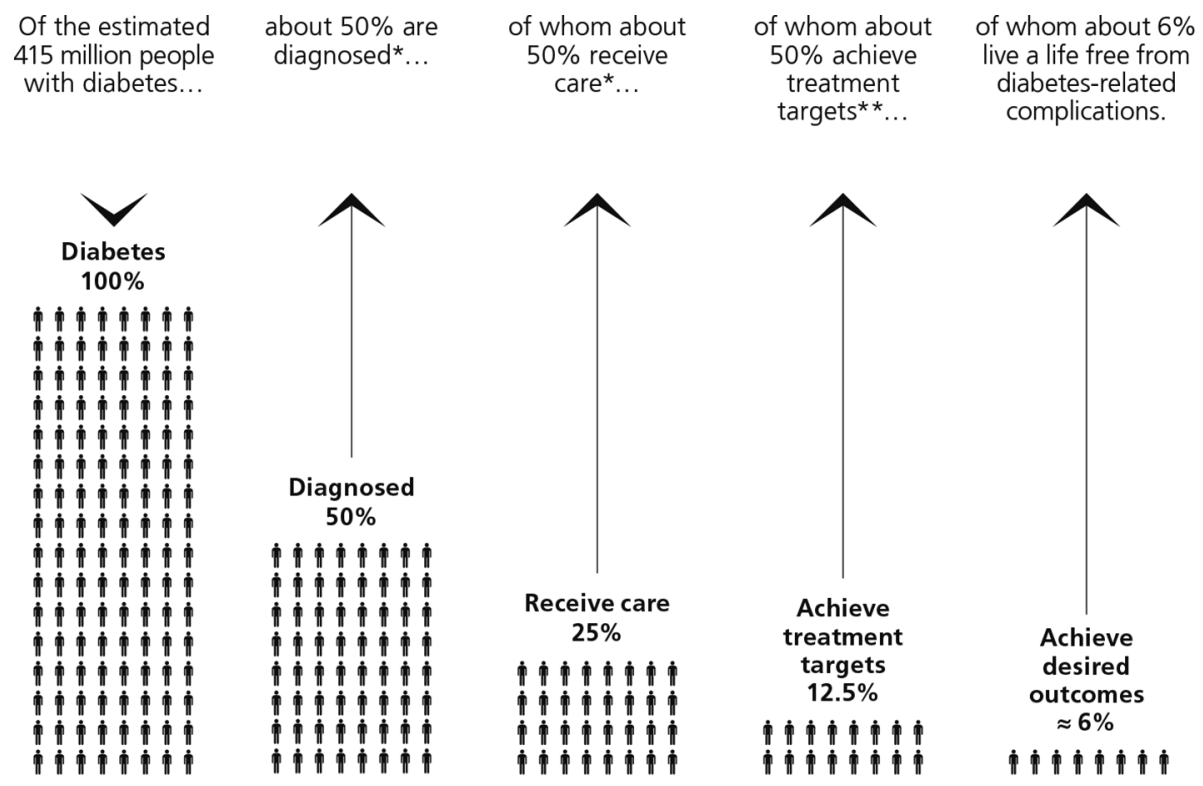

Figure 2 The Rule of Halves theoretical framework. ${ }^{12}$ 


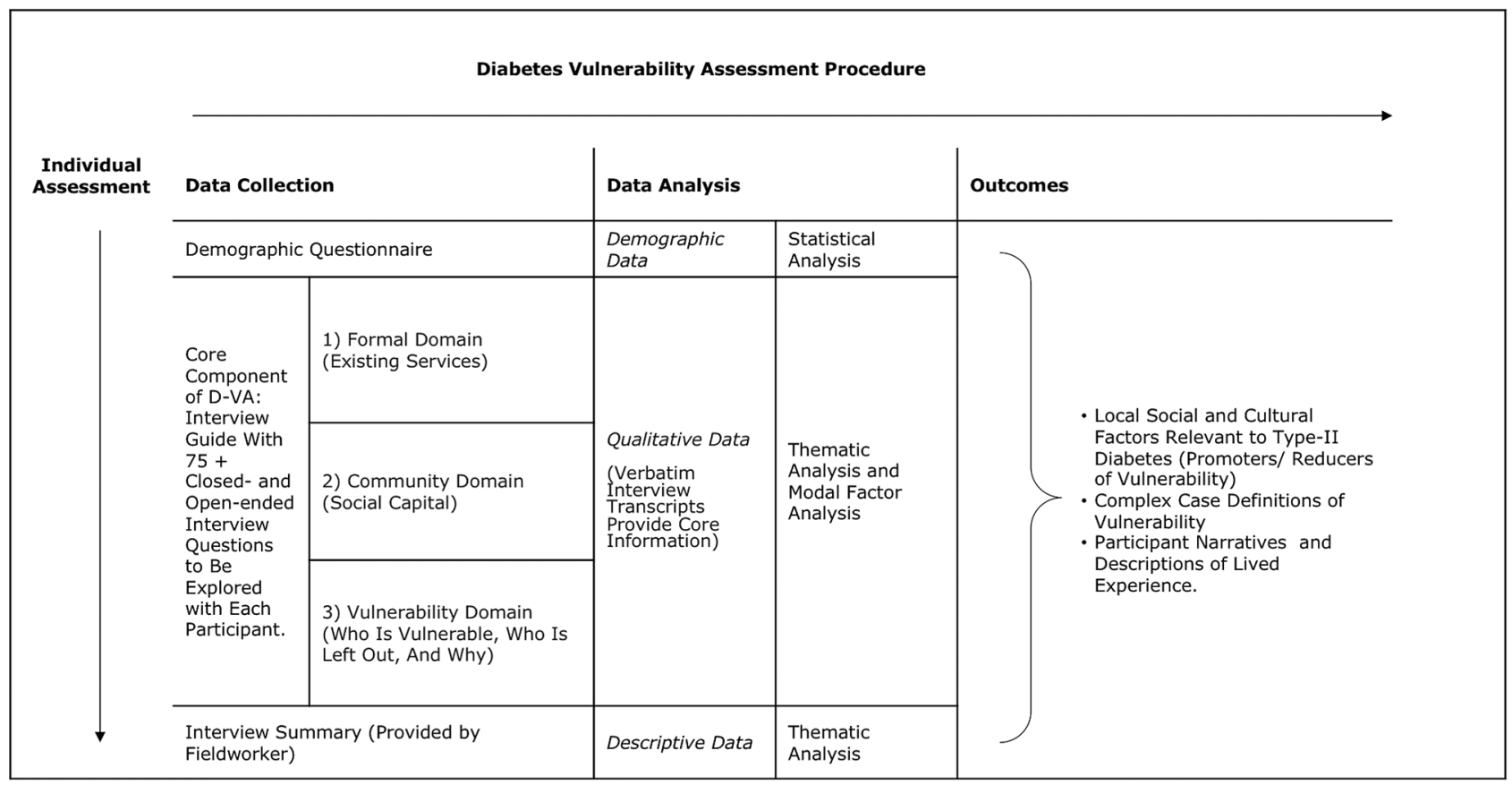

Figure 3 The Diabetes Vulnerability Assessment (D-VA) procedure.

of adaptation and resilience) and a vulnerability domain (assessing barriers to individual capability and opportunity). This structure allows for replication in various settings, yielding the growing global database for vulnerability factors in diabetes.

\section{Local D-VA}

In each city, based on local and global group consensus, a target group for the VA is identified: either people diagnosed with type 2 diabetes and/or people at increased risk of developing type 2 diabetes.

\section{Participant selection}

Participants are recruited locally employing a purposive sampling technique. In line with qualitative research practices, such sampling makes possible the capturing of salient insights from target populations. Based on local and global team consensus, so-called case filters (eg, high BMI, living alone, living in a community without access to health insurance) 'flag' individuals in the local population to be contacted for participation. In the first five cities, case filters were generated through statistical means (Houston) or were directly derived from existing data (Copenhagen). In others, where available data proved insufficient, global and local academic teams agreed on case filters for recruitment based on new research (Mexico City) or recruitment through community health centres and tertiary care facilities (Shanghai and Tianjin).

\section{Application of the assessment and field worker training}

In each city, the D-VA is tailored to the knowledge base and research capabilities of local academic partners and revised in accordance with local language use and related health service and community structures. Data collection is carried out following the city-specific
D-VA, which includes an interview guide consisting of approximately 75 closed and open-ended questions. The in-depth semistructured individual interviews are conducted by experienced field workers representing the local academic partner and trained by UCL in multiday workshops. Short pilots precede the data collection phase so as to allow for modifications of the interview protocol where needed. During data collection, field workers are discouraged from taking extensive notes so as to establish a rapport with participants. All interviews are tape recorded, transcribed verbatim, and coded for analysis.

\section{Data analysis strategies}

Data for analysis include verbatim interview transcripts (primary data) and basic demographic and biomedical information recorded for each interviewee. Furthermore, executive summaries of each interview with ethnographic observations and comments are drawn up by field workers (secondary data), and these are translated where necessary. Executive summaries are based on a global template developed by UCL. So far, a total of 746 individual in-depth interviews have been conducted across the first five cities.

Primary data are coded and analysed by the specific local academic partner applying similar methodologies: Systematic Text Condensation ${ }^{20}$ in Copenhagen, Grounded Theory ${ }^{21}$ in Mexico City and Thematic Content Analysis (TCA) ${ }^{22-24}$ in Houston, Tianjin and Shanghai identifying relevant local social factors and cultural determinants that impact how health and well-being are experienced among individuals at local levels. The local methodologies will be presented in detail separately elsewhere. 
Secondary data are coded by UCL using TCA and with input from the respective local academic partner to analyse social factors and cultural determinants across cities. The global analysis runs along three parallel and complimentary trajectories. The first trajectory focuses on collating and analysing relevant local data sets following well-established TCA principles, making possible the identification of globally salient themes. The second trajectory focuses on synthesising the local findings into a set of social factors and cultural determinants of diabetes that goes across the cities. In this way, a repository of social factors and cultural determinants-locally and across cities- is established, which will evolve continuously as further data from additional cities are included. The third trajectory focuses on the development of locally useful case definitions of vulnerability that build on combinations of globally recognisable factors and local circumstances. These unique permutations of combined factors help guide local policy-makers in the application of resources for public health, urban planning and clinical and social services.

\section{ETHICS AND DISSEMINATION}

The research in each city is conducted in accordance with the ethical principles of the Declaration of Helsinki. Informed written consent is obtained from participants. The respondents are anonymised and the personal data are only known to and protected by local academic partners. Findings are presented in aggregated form. In addition, precautions are taken to ensure that no respondents are identifiable. As a regulatory requirement of Novo Nordisk, safety information from a participant relating to a Novo Nordisk product is reported within 24 hours.

The dissemination strategy of the research focuses on building bridges between participating cities and on sharing results with both academic and policy stakeholders.

- The first Global Cities Changing Diabetes Summit was held in Copenhagen in November 2015. Presentations and urban diabetes study results are available online. A second Global Summit took place in October 2017.

- The global research findings will be published separately in peer-reviewed journals and presented at international conferences.

- The local research results from each city will be published separately in peer-reviewed national and international journals and presented at national and international conferences.

Acknowledgements The authors wish to thank the following local academic partner institutions, as well as the academic leads and their dedicated field workers in each of the five cities for conducting local D-VAs: National Institute of Public Health of Mexico, led by Dr Simón Barquera; University of Copenhagen, Department of Public Health, led by Professor Finn Diderichsen; University of Texas, School of Public Health, led by Professor Stephen H Linder; Tianjin Medical University, School of Public Health, led by Professor Jun Ma; Shanghai Jiao Tong University, Shanghai Diabetes Institute, led by Professor Weiping Jia.
Contributors ADN is lead investigator and global academic lead for the study programme; responsible for the research framework and study design. He is content expert on sociocultural factors and on vulnerability. He collaborates with and provides guidance to the local academic leads and training of local fieldworkers, and will be carrying out the qualitative global analysis and interpretation. JJN is coinvestigator and content expert on the biomedical aspects of diabetes, diabetes management and care pathways. He contributes to the global research framework and provides critical input on the programme's quantitative research and research dissemination. MB and LH are coinvestigators. They contribute to the global research framework and study design through project management and content support. They will also be supporting the data collection and providing input to the research parameters. They are responsible for the dissemination plan and helped to prepare the initial draft manuscript. AMV is coinvestigator and method expert for qualitative and mixedmethod approaches to health research. She contributes to the global research framework and study design, supports the local academic teams and training of local fieldworkers and will be carrying out the qualitative global analysis and interpretation.

Funding Novo Nordisk has committed to fund US\$20 million towards 2020. Competing interests None declared.

Ethics approval Local approval was granted by relevant bodies in each participating city. These were: Danish Data Protection Agency (Copenhagen); National Institute of Public Health (INSP) Ethics Committee (Mexico City); Ethics Committee of Shanghai People's Hospital (Shanghai); Ethics Committee of the Tianjin Medical University (Tianjin); IntegReview IRB (Houston); Research Ethics Board University of British Columbia (Vancouver).

Provenance and peer review Not commissioned; externally peer reviewed.

Open Access This is an Open Access article distributed in accordance with the Creative Commons Attribution Non Commercial (CC BY-NC 4.0) license, which permits others to distribute, remix, adapt, build upon this work non-commercially, and license their derivative works on different terms, provided the original work is properly cited and the use is non-commercial. See: http://creativecommons.org/ licenses/by-nc/4.0/

(c) Article author(s) (or their employer(s) unless otherwise stated in the text of the article) 2017. All rights reserved. No commercial use is permitted unless otherwise expressly granted.

\section{REFERENCES}

1. International Diabetes Federation. IDF Diabetes Atlas. 7th edn. Belgium: Brussels, 2015.

2. United Nations Department of Economic and Social Affairs (UNDESA). World urbanization prospects, the 2014 revision, highlights, 2014

3. Alirol E, Getaz L, Stoll B, et al. Urbanisation and infectious diseases in a globalised world. Lancet Infect Dis 2011;11:131-41.

4. Allender S, Foster C, Hutchinson L, et al. Quantification of urbanization in relation to chronic diseases in developing countries: a systematic review. J Urban Health 2008;85:938-51.

5. Galea S, Vlahov D. Urban health: evidence, challenges, and directions. Annu Rev Public Health 2005;26:341-65.

6. ICSU. Report of the ICSU planning group on health and wellbeing in the changing urban environment: a systems analysis approach: International Council for Science, Paris, 2011.

7. Dagenais GR, Gerstein HC, Zhang X, et al. Variations in diabetes prevalence in low-, middle-, and high-income countries: results from the prospective Urban and Rural Epidemiological study. Diabetes Care 2016;39:780-7.

8. Marmot M. Social determinants of health inequalities. Lancet 2005;365:1099-104.

9. Marmot M, Wilkinson R. Social determinants of health. 2nd ed. Oxford: Oxford University Press, 2005.

10. Farmer PE, Nizeye B, Stulac S, et al. Structural violence and clinical medicine (Policy Forum). PLoS Med 2006;3:e449.

11. World Health Organization. What are social determinants of health? 2015 http://www.who.int/social_determinants/sdh_definition/en/.

12. Hart JT. Rule of halves: implications of increasing diagnosis and reducing dropout for future workload and prescribing costs in primary care. Br J Gen Pract 1992;42:116-9.

13. Smith WC, Lee AJ, Crombie IK, et al. Control of blood pressure in Scotland: the rule of halves. BMJ 1990;300:981-3. 
14. Wilkerson HL, Krall LP. Diabetes in a New England town: a study of 3,516 persons in Oxford, Mass. J Amer Med Assoc 1947;135:209-16.

15. Sharp C. Diabetes survey in Bedford 1962. P Roy Soc Med 1964;57:193-202.

16. Doney BJ. An audit of the care of diabetics in a group practice. J Roy Coll Gen Pract 1976;26:734-42.

17. Wilkes E, Lawton EE. The diabetic, the hospital and primary care. J R Coll Gen Pract 1980;30:199-206.

18. Mufunda J, Ghebrat $\mathrm{Y}$, Usman A, et al. Underestimation of prevalence of raised blood sugar from history compared to biochemical estimation: support for the WHO rule of halves in a population based survey in Eritrea of 2009. Springerplus 2015;4:1-5.

19. The Tripartite Core Group. Post-Nargis periodic review I: the Association of Southeast Asian Nations (ASEAN), the government of the union of Myanmar and the United Nations, 2008.
20. Malterud K. Systematic text condensation: a strategy for qualitative analysis. Scand J Public Health 2012;40:795-805.

21. Strauss A, Corbin J. Grounded theory methodology. In: Denzin NK, Lincoln YS, eds. Handbook of qualitative research. Thousand Oaks, CA: SAGE Publications, 1994:273-85.

22. Boyatzis RE. Transforming qualitative information: thematic analysis and code development. Thousand Oaks, CA: SAGE Publications, 1998.

23. Braun V, Clarke V. Using thematic analysis in psychology. Qual Res Psychol 2006;3:77-101.

24. Fereday J, Muir-Cochrane E. Demonstrating rigor using thematic analysis: A hybrid approach of inductive and deductive coding and theme development. Int J Qual Methods 2006;5:80-92. 